\title{
Focusing Light through Turbid Media by Binary Amplitude Shaping
}

\author{
D. Akbulut, E.G. van Putten, W.L. Vos, and A.P. Mosk
}

Complex Photonic Systems, MESA+ Research Institute and Dept. of Science \& Technology, University of Twente, Enschede, The Netherlands 
Many materials such as paint, human skin, egg shell and paper are turbid materials which scatter light strongly. If coherent light is transmitted through such materials, a speckle pattern is formed. Here, we demonstrate that light is focused to a bright spot after such a material by selectively blocking parts of the light wave entering the material. By this method, it is possible to utilize fast, MEMS-based binary amplitude modulators for wavefront shaping. This method could prove useful for applications in medical imaging.
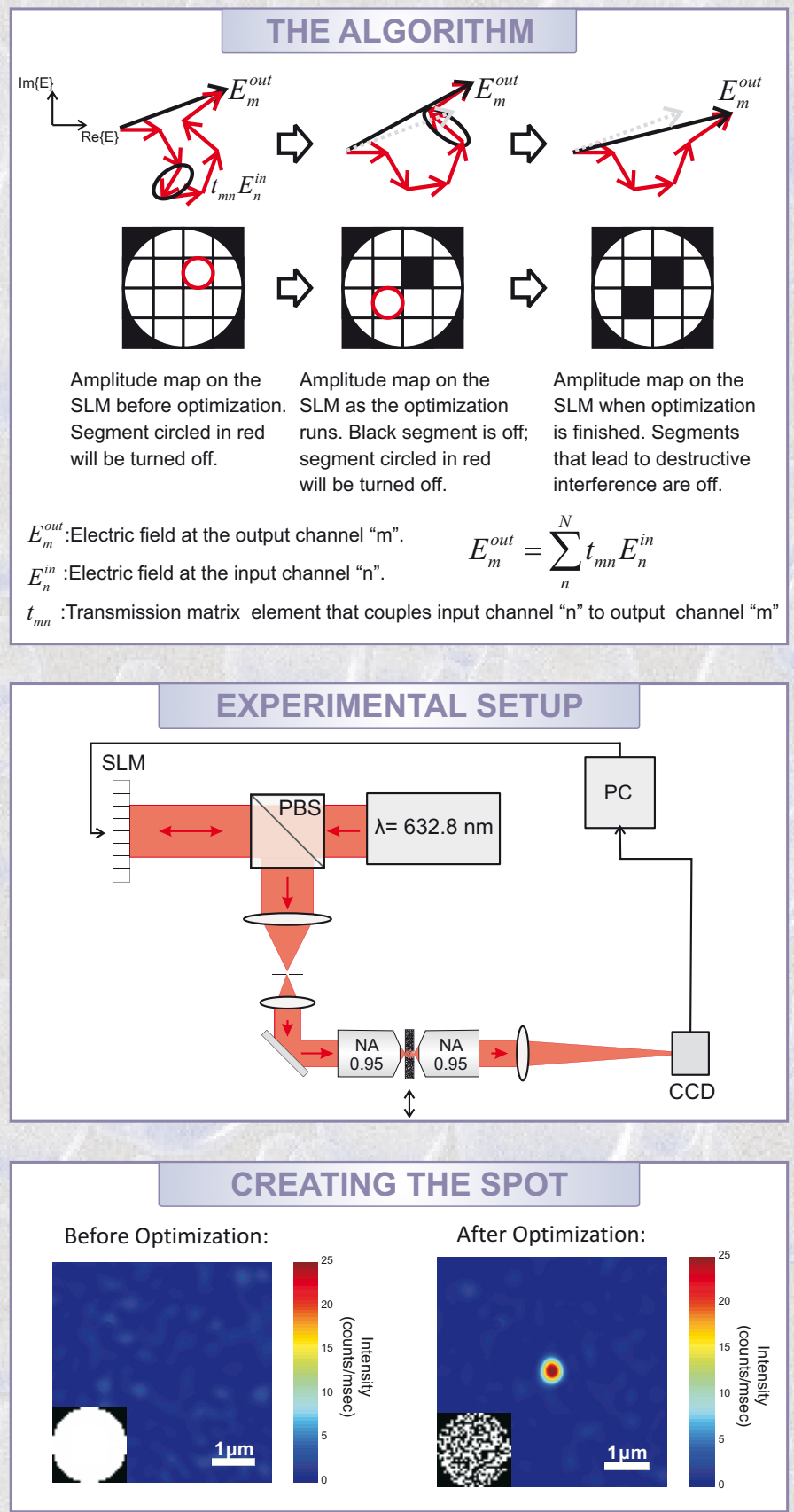

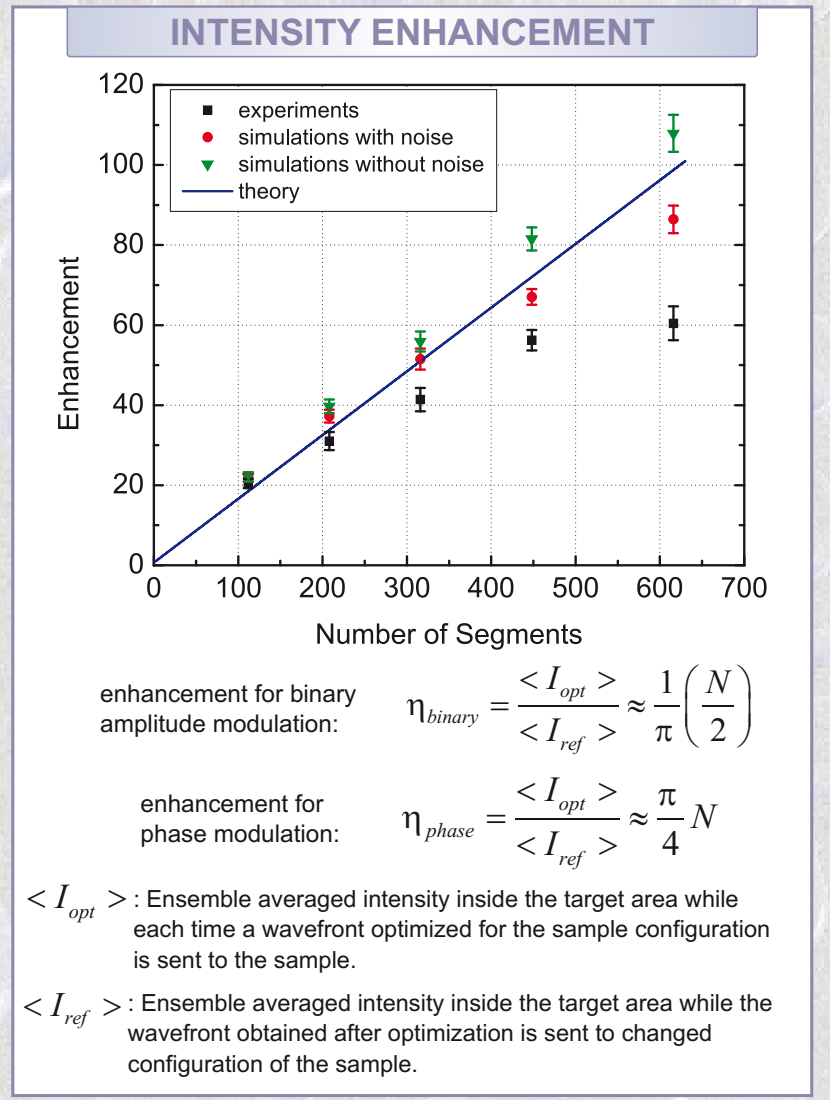

\section{CONCLUSIONS}

We have demonstrated focusing light through a strongly scattering material by blocking part of the light field entering the sample.

The intensity of light at the target position is enhanced by a factor of $\sim 60$ when SLM is divided into $\sim 600$ segments.

-I.M. Vellekoop and A.P. Mosk, "Focusing coherent light through opaque strongly scattering media" Optics Letters, 32, 2309-2311 (2007)

-I.M. Vellekoop and A.P. Mosk, "Phase control algorithms for focusing light through turbid media" Optics Communications, 281, 3071-3080 (2008) 